\title{
Palliative Care in der Schweiz 2008 - eine Bestandesaufnahme
}

Steffen Eychmüller ${ }^{a}$,

Margareta Schmida, ,

Marianne Müller ${ }^{b}$

a Palliativzentrum, Kantonsspital St. Gallen

b Züricher Hochschule für angewandte Wissenschaften ZHAW

c Institut für Sozialund Präventivmedizin, Universität Zürich
Interessensverbindung:

Der Erstautor war von 2005 bis 2009 Co-Präsident der Schweizerischen Gesellschaft für Palliative Medizin, Pflege und Begleitung «palliative ch».

Sponsoring:

Dieses Projekt wurde durch eine Forschungsförderung der Oncosuisse ermöglicht.

Korrespondenz: Dr. med. Steffen Eychmüller FMH Innere Medizin Palliativzentrum Kantonsspital St. Gallen CH-9007 St. Gallen Tel. 0714943550 Fax 0714946255 steffen.eychmueller@kssg.ch

\section{Hintergrund}

Die Veränderung der demographischen Entwicklung mit einem immer grösseren Anteil alter Menschen, der Zunahme von chronischen Erkrankungen und dem raschen Anstieg der Sterberate stellt die Planung im schweizerischen Gesundheitswesen vor eine riesige Herausforderung. Immer mehr Menschen werden in den kommenden Jahrzehnten in der Schweiz versterben, wenn die zahlenmässig starke «Babyboom»-Generation in die letzte Lebensphase kommt. Bis zum Jahr 2050 rechnet das Bundesamt für Statistik [1] mit einem Anstieg der Sterberate um 50\% von derzeit etwas mehr als 60000 Todesfällen pro Jahr auf über 90000. Zudem zeichnet sich ein Mangel an Pflegepersonal ab [2].

Derzeit versterben $80 \%$ aller Menschen in der Schweiz in Spitälern oder Pflegeheimen [3]. Rund 10\% aller Gesundheitsausgaben fallen in den letzten sechs Monaten eines Menschenlebens an [4]. Bereits in den kommenden Jahrzehnten werden sehr hohe Kosten auf unsere Gesellschaft zukommen, wird der gegenwärtige Weg der Versorgung schwerkranker und sterbender Menschen beibehalten. Die Diskussionen um die Mittelzuteilung, die Triagierung, Fragen zur Indikationsqualität, aber auch die Diskussion um die Sterbehilfe im Zeitalter der Rationierung medizinischer und pflegerischer Leistungen werden deshalb immer drängender und vehementer. Neue Formen der Betreuung von chronisch Kranken, Schwerkranken und Sterbenden sind deshalb dringend erforderlich [4]. Ist die Schweiz für diese Herausforderung gerüstet? Wie steht es um die Palliative-Care-Angebote in der Schweiz, und wie ist der Zugang der Bevölkerung zu diesen Leistungen?

\section{Entwicklung der Palliative Care in der Schweiz}

In einer ersten Bestandesaufnahme aus der Schweiz aus dem Jahr 2001 [5] zeigte sich, dass das Angebot an spezialisierter Palliative Care in der Schweiz regional extrem unterschiedlich war und ein grosser Bedarf an Klärung sowohl minimaler Definitionen als auch von Qualitäts- und Ausbildungsstandards bestand. Bei einer im Jahr 2006 veröffentlichten Studie zum Angebot der Palliative Care in 38 Ländern Europas [6] fällt die Schweiz durch einen sehr hohen Anteil an «No data»-Antworten auf. Weder zur Anzahl von Palliativteams noch zu Spitälern mit Palliativabteilungen oder zu Hospizen gab es klare Angaben.

\section{Tour d'horizon des soins palliatifs}

\section{en Suisse en 2008}

En Suisse, de plus en plus de gens décéderont au cours de ces prochaines décennies lorsque la génération nombreuse née du baby-boom arrivera dans sa dernière phase de vie. L'OMS considère les soins palliatifs comme une stratégie primordiale pour relever cet immense défi.

L'enquête sur la situation des soins palliatifs en Suisse montre qu'il existe des lacunes importantes en matière de définition des prestations, d'information, de qualifications professionnelles et de disponibilité des offres entre les différentes régions du pays. Cette étude servira de base aux développements futurs nouvellement définis par la Confédération et les cantons dans la «Stratégie nationale en matière de soins palliatifs 2010-2012».

Die vorliegende Bestandesaufnahme soll aufzeigen, wie weit die Palliativ-Versorgung in medizinischer, pflegerischer und psychosozialer Hinsicht in der Schweiz in der Grundversorgung in Spitälern, in Alters- und Pflegeheimen, im ambulanten Sektor wie auch in Spezialeinrichtungen für Palliative Care entwickelt ist, und wie die Akteure die Situation der Versorgung aktuell und künftig einschätzen.

\section{Methodik}

Die Erhebung baute auf fünf Schritten auf: Nach einem Screening relevanter internationaler und nationaler Datenbanken und Dokumente (Schritt 1) wurden Fragestellungen und Kriterien zur Entwicklung von Palliative Care als inhaltliche Grundlage für den Fragebogen definiert (Schritt 2). Anschliessend (Schritt 3) erfolgte die Entwicklung des Befragungsinstrumentes, was aufgrund der unterschiedlichen zu befragenden Institutionen eine besondere Herausforderung darstellte (Pflegeheime, Spitäler, Spitex usw.). Nach zwei Pretestphasen wurde der definitive Fragebogen Ende 2007 in die französische Sprache über- 
setzt. Aufgrund der kleinen Stichprobe aus der italienischen Schweiz wurde auch dort der französische Fragebogen verwendet.

Der Fragebogen enthielt gesamthaft 122 Fragen aus fünf verschiedenen Bereichen; neben Detailangaben zum Angebot an palliativspezifischen Leistungen wurden auch die Einschätzung von Palliative Care im Allgemeinen (Brisanz, Image, Definition) und das Vorgehen bei der Betreuung von Menschen mit einer progredienten, weit fortgeschrittenen Krankheit und begrenzter Lebenserwartung erfasst.

Aufgrund von Adresslisten des Spitalverbandes H+ $(n=315)$, der Curaviva $(n=1469)$ sowie der spezifischen spitalexternen onkologischen und mobilen Palliative-Care-Dienste wurden in diesen Institutionen Vollerhebungen durchgeführt (Schritt 4). Aus allen vorhandenen Adressen von Spitexorganisationen wurde eine nach Kanton stratifizierte Zufallsstichprobe vom Umfang 300 gezogen.

Zur Datenerfassung (Schritt 5a) wurde das Programm Epidata Version 3.1. (www.epidata.dk) verwendet. Für die statistische Auswertung (Schritt 5b) wurden die Daten ins Statistikprogramm SPSS Version 14 importiert und damit deskriptiv ausgewertet. Für bestimmte Fragestellungen wurde die gleichzeitige Beantwortung mehrerer Fragen mit Hilfe von Kreuztabellen analysiert.

\section{Ergebnisse}

Im März 2008 wurden gesamthaft 2115 Fragebogen (1594 d/521 f) an die Pflegedienstleitungen der Schweizer Spitäler, Heime, spezialisierten PalliativeCare-Institutionen und mobilen Palliative-CareDienste sowie der Spitex geschickt.

Der Rücklauf betrug 57\%, entsprechend 1195 Fragebogen. Drei Viertel der Antworten (881) stammten aus der deutschen Schweiz und ein Viertel (314) aus der französischen und italienischen Schweiz. $65 \%$ der Antworten entfielen auf Alters- und Pflegeheime, 15\% auf die Spitäler und 20\% auf mobile Dienste.

\section{Meinungsbild zur Palliative Care in der Schweiz}

\section{Bekanntheit von Palliative Care aus Sicht der Befragten}

Fast 50\% der teilnehmenden Pflegedienstleitenden waren der Meinung, dass die Definition in der Öffentlichkeit nicht oder eher nicht klar sei, und 36\% waren teilweise dieser Meinung. Die Unterschiede zwischen den Institutionstypen sind klein. Die Meinung zum Wissen über das Palliative-Care-Angebot in der Öffentlichkeit ist noch eindeutiger: $64 \%$ der Befragten waren der Ansicht, dass dieses Wissen eher schlecht oder schlecht sei.

\section{Politische Unterstützung durch den Kanton}

Ungefähr die Hälfte der Antwortenden (zwischen 43\% bis $52 \%$ je nach Institutionstyp) war der Meinung, dass die politische Unterstützung für Palliative Care in ihrem Kanton zu klein sei. Nur knappe $20 \%$ beurteilten sie als gerade richtig, wobei die Spitäler die politische Unterstützung etwas positiver beurteilten als die Heime und spitalexternen Dienste.

\section{Image und «Marktwert» von Palliative Care}

Fast die Hälfte der Befragten in den Heimen und den spitalexternen und mobilen Diensten war der Meinung oder eher der Meinung, Palliative Care werde zunehmend zu einem Marketingfaktor in der Gesundheitsversorgung. Die Spitäler waren dabei etwas skeptischer und nur ein Drittel stimmte dieser Einschätzung $\mathrm{zu}$. Weitere rund 30\% der Befragungs-Teilnehmenden unterstützten teilweise diese Meinung.

\section{Verankerung von Palliative Care im Leitbild - Ist, Soll und Umsetzung}

Alters- und Pflegeheime waren überwiegend der Meinung, dass Palliative Care ein fester Bestandteil des Leitbilds sein sollte (90\% gegenüber $68 \%$ bei den Spitälern). Fragt man nach der tatsächlichen Verankerung, dann war Palliative Care aber nur noch bei 50\% der befragten Alters- und Pflegeheime tatsächlich im eigenen Leitbild verankert (Spitäler 25\%). Schaut man weiter die konkrete Umsetzung an, zum Beispiel am Vorhandensein eines spezifischen interdisziplinären Rapport- und Kommunikationssystems, so war dies in allen Institutionsarten einschliesslich der mobilen Dienste nur noch in ca. $25 \%$ Realität.

\section{Palliativspezifisches Vorgehen in der Grundversorgung}

\section{Anwendung palliativspezifischer Standards und Fachqualifikation}

In Anbetracht der grossen Zahl an schwerkranken und sterbenden Menschen in den Institutionen ist palliativspezifisches Vorgehen in der Grundversorgung bisher eher wenig verbreitet, wie die nachfolgende Graphik (Abb. 1) zeigt.

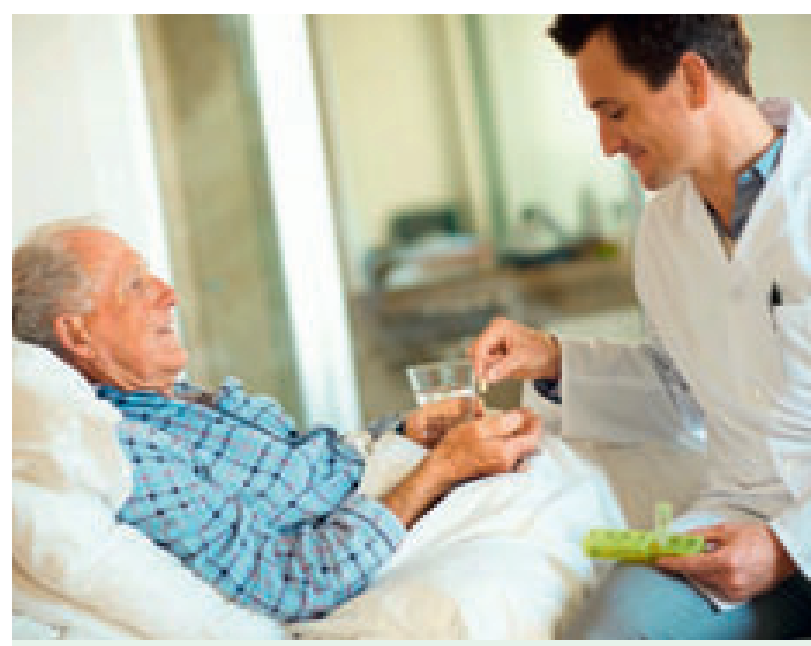

Wie ist der Stand der palliativen Versorgung von Patienten in der Schweiz? 


\section{Entscheidungsfindung, Einbezug} von Patientinnen, Patienten und Angehörigen und Betreuung in der Sterbephase

Geht es um den regelmässigen Einbezug von Patientinnen, Patienten und Angehörigen bei der Festlegung von Behandlungszielen und Massnahmen (Standortgespräche), so ist dies etwas weniger häufig in Spitälern (70\%) als in Alters- und Pflegeheimen bzw. spitalexternen und mobilen Diensten (78\% bzw. $81 \%$ ).

Betrachtet man die Sterbe- und Trauerphase, so zeigen sich markante Unterschiede zwischen Heimen und Spitälern. Die Alters- und Pflegeheime geben viel häufiger an, Patienten und Angehörige auf die Patentenverfügung anzusprechen (82\%), persönliche Anliegen der Patienten bezüglich Sterben und Todesfall zu besprechen und zu dokumentieren (92\%) und Möglichkeiten zum Abschiednehmen anzubieten (92\%). Die spitalexternen Dienste liegen etwas darunter, bieten diese Möglichkeiten aber ebenfalls häufiger an als die Spitäler. Noch weit weniger ausgebaut ist die $\mathrm{Zu}-$ sammenarbeit mit freiwilligen Helferinnen und Helfern. Auch hier ist der Anteil in den Alters- und Pfle-

\section{Abbildung 1}

Anwendung palliativspezifischer Standards in der Grundversorgung

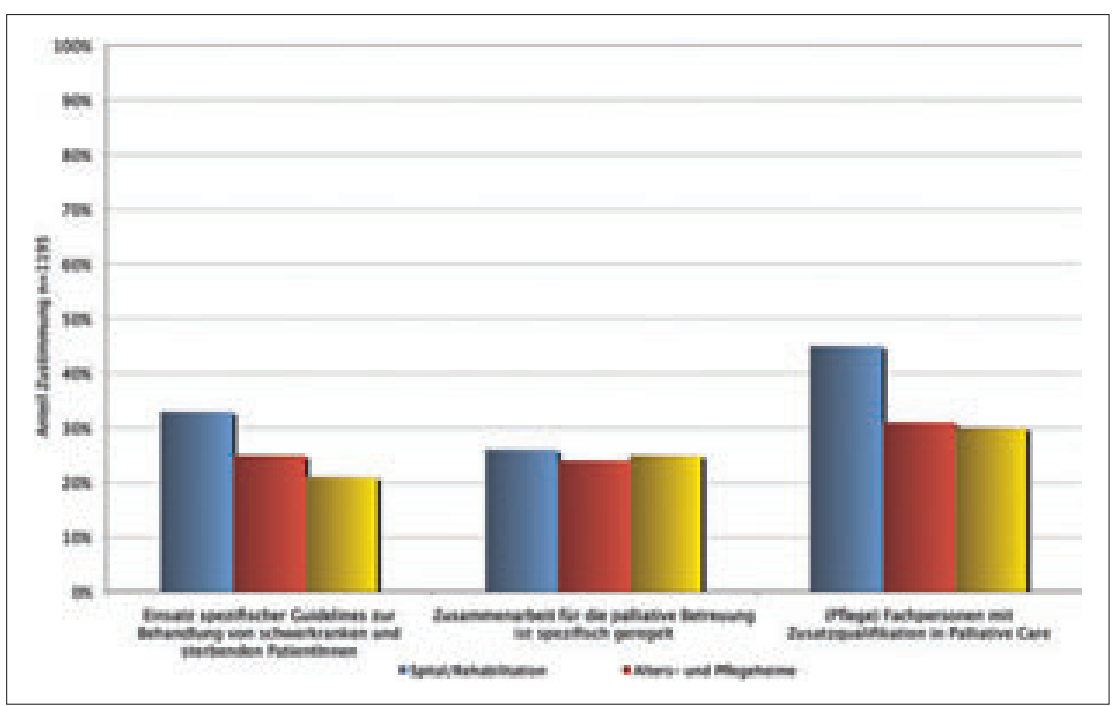

Tabelle 1

Zusammenarbeit mit dem regionalen Palliativ-Netz

\begin{tabular}{lclllc}
$\begin{array}{l}\text { Regionales } \\
\text { Palliativnetz }\end{array}$ & $\begin{array}{l}\text { ist fester } \\
\text { Bestandteil } \\
\text { des Netzes }\end{array}$ & $\begin{array}{l}\text { ist im } \\
\text { Austausch } \\
\text { mit dem } \\
\text { Netz }\end{array}$ & $\begin{array}{l}\text { es gibt } \\
\text { kein } \\
\text { Netz }\end{array}$ & $\begin{array}{l}\text { nicht bekannt, } \\
\text { ob es } \\
\text { regionales } \\
\text { Netz gibt }\end{array}$ & $\begin{array}{l}\text { fehlende } \\
\text { Antwort }\end{array}$ \\
$\begin{array}{l}\text { Spital/ } \\
\text { Rehabilitation }\end{array}$ & $14 \%$ & $39 \%$ & $6 \%$ & $10 \%$ & $19 \%$ \\
$\begin{array}{l}\text { Alters- und } \\
\text { Pflegeheime }\end{array}$ & $5 \%$ & $29 \%$ & $9 \%$ & $26 \%$ & $6 \%$ \\
$\begin{array}{l}\text { Spitex, mobiler } \\
\text { Dienst, anderes }\end{array}$ & $17 \%$ & $30 \%$ & $11 \%$ & $19 \%$ & $8 \%$ \\
\hline \begin{tabular}{l} 
Total \\
\hline
\end{tabular} & $9 \%$ & $31 \%$ & $9 \%$ & $22 \%$ & $9 \%$
\end{tabular}

geheimen mit $45 \%$ und bei den spitalexternen Diensten mit $60 \%$ grösser als in den Spitälern (36\%).

\section{Vernetzung in der Palliativversorgung}

Eine der häufigsten Klagen sowohl seitens der Patienten als auch seitens der Akteure im Gesundheitswesen sind die Mängel bei der Vernetzung der Leistungen. Hausärztinnen und -ärzte beklagen die oft mangelnde Information über Entscheide und z. B. unnötige Änderung der Medikation durch die Spitäler. Palliativnetze könnten zur Verbesserung dieser Mängel beitragen. Doch ihre Existenz ist häufig nicht bekannt (22\%) und die regelmässige Kooperation ist mit $9 \%$ sehr gering (Tabelle 1).

\section{Spezialisierte Angebote der Palliative Care -} Spezialeinrichtungen und Spazialabteilungen Von den gesamthaft antwortenden Institutionen ( $n=1195)$ konnten aufgrund einer Selbsteinschätzung durch die Verantwortlichen der Institutionen 138 Einrichtungen oder Organisationen mit spezialisiertem Angebot in Palliativ Care identifiziert und 130 einem Bereich zugeordnet werden. Auf den Langzeitbereich entfallen hiervon 48 Institutionen, 41 auf den Akutbereich (stationär) und 30 auf den mobilen Bereich. 11 Institutionen bieten sowohl Leistungen der Akut- als auch der Langzeitversorgung.

\section{Spezialisierte Palliative Care zwischen}

\section{Anspruch und Wirklichkeit}

Spezialisierte Palliative Care ist international an Kriterien geknüpft wie die regelmässige Verfügbarkeit eines interprofessionellen Palliative Care Teams mit fachspezifischer Ausbildung, an eine geregelte Zusammenarbeit, sowie zum Beispiel an den Einsatz palliativspezifischer Symptomerfassungs-Instrumente und Behandlungsleitfäden.

Institutionen, die in der vorliegenden Befragung für sich den Status «spezialisiert» in Anspruch nehmen, erfüllen derartige Kriterien und Anforderungen nur teilweise. Auffällig ist hierbei, dass die medizinische Kernkompetenz im Team (Ärztin/Arzt) ausserhalb der Spitäler nur sehr wenig integriert ist (im Langzeitbereich in $50 \%$, ausserhalb einer Institution/mobile Dienste nur in 37\%; Tabelle 2).

Zwar weisen Institutionen, welche das Gütesiegel «spezialisiert in Palliative Care» für sich in Anspruch nehmen, einen höheren Prozentsatz an palliativspezifischen Vorgehensweisen auf (z. B. Einsatz spezifischer Behandlungsguidelines; Tabelle 3), aber insgesamt zeigen diese Zahlen eine vergleichsweise geringe Verbreitung palliativspezifischen Vorgehens in der Schweiz.

Auch das Kriterium der 24-Stunden-Erreichbarkeit einer Fachperson in Palliative Care («Hintergrunddienst») wird von den spezialisierten Institutionen für Palliative Care nur teilweise erfüllt. Nur gerade ein Viertel der Institutionen im Langzeitbereich und im Spital verfügt über einen 24-Stunden-Hintergrunddienst. Findet die Versorgung ausserhalb einer Insti- 
Tabelle 2

Interprofessionelle Zusammensetzung spezialisierter Palliativteams (Selbstdeklaration)

\begin{tabular}{|c|c|c|c|c|c|c|c|c|}
\hline $\begin{array}{l}\text { Palliative } \\
\text { Care Team }\end{array}$ & $\begin{array}{l}\text { Ärztin/ } \\
\text { Arzt }\end{array}$ & Pflege & $\begin{array}{l}\text { Psycho- } \\
\text { logie }\end{array}$ & $\begin{array}{l}\text { Sozial- } \\
\text { arbeit }\end{array}$ & $\begin{array}{l}\text { Physio- } \\
\text { therapie }\end{array}$ & Pfarrer & $\begin{array}{l}\text { Trauer- } \\
\text { begl. }\end{array}$ & $\begin{array}{l}\text { Frei- } \\
\text { willige }\end{array}$ \\
\hline Langzeitbereich & $50 \%$ & $85 \%$ & $17 \%$ & $17 \%$ & $31 \%$ & $60 \%$ & $23 \%$ & $73 \%$ \\
\hline Akutspital & $81 \%$ & $88 \%$ & $46 \%$ & $54 \%$ & $66 \%$ & $68 \%$ & $20 \%$ & $37 \%$ \\
\hline $\begin{array}{l}\text { Ausserhalb } \\
\text { einer Institution }\end{array}$ & $37 \%$ & $70 \%$ & $10 \%$ & $17 \%$ & $13 \%$ & $10 \%$ & $30 \%$ & $37 \%$ \\
\hline $\begin{array}{l}\text { Akut- und Lang- } \\
\text { zeitbereich }\end{array}$ & $73 \%$ & $82 \%$ & $36 \%$ & $18 \%$ & $46 \%$ & $36 \%$ & $36 \%$ & $54 \%$ \\
\hline Total & $57 \%$ & $83 \%$ & $26 \%$ & $27 \%$ & $40 \%$ & $51 \%$ & $25 \%$ & $50 \%$ \\
\hline
\end{tabular}

\section{Tabelle 3}

Einsatz spezifischer Guidelines zur Behandlung von schwerkranken und sterbenden Patienten

\begin{tabular}{lllllll} 
& \multicolumn{2}{l}{ Alle Institutionen } & & \multicolumn{2}{l}{ Spezialeinrichtungen } \\
\hline Einsatz spezifischer Guidelines & Ja & Nein & fehlend & Ja & Nein \\
\hline Spital/Rehabilitation & $33 \%$ & $47 \%$ & $20 \%$ & $67 \%$ & $33 \%$ \\
\hline Alters- und Pflegeheime & $25 \%$ & $68 \%$ & $7 \%$ & $48 \%$ & $52 \%$ \\
\hline Spitex, mobiler Dienst, anderes & $21 \%$ & $69 \%$ & $10 \%$ & $64 \%$ & $36 \%$
\end{tabular}

tution statt, so steht ein solcher Dienst mit einem Anteil von $53 \%$ ebenfalls nicht in genügendem Ausmass zur Verfügung.

\section{Diskussion}

Palliative Care als Vorgehenskonzept für schwerkranke und sterbende Menschen scheint aus Sicht der Leistungsanbieter bisher in der Schweizerischen Bevölkerung wenig bekannt zu sein. Auch wenn solche Zahlen für die Schweiz fehlen, ist davon auszugehen, dass ein Grossteil der Menschen hier nicht genügend über Palliative Care und die Angebote informiert ist $[7,8]$.
Einordnung des Leistungsniveaus der eigenen Institution. Nimmt man internationale Vergleiche vor [6], so ist die Schweiz sowohl hinsichtlich Anzahl der Institutionen als auch der stationären Betten für Palliative Care wenig entwickelt. Bei der Interpretation der Daten bleibt jedoch ein grosses Unbehagen: $\mathrm{Zu}$ unscharf ist das Profil der einzelnen Angebote aufgrund fehlender gemeinsamer Definitionen von Leistungen und Leistungsniveaus der Palliative Care. So lange solche Rahmendefinitionen fehlen, können viele Anbieter heute mit guter Begründung sagen, dass sie Palliative Care «machen».

Insbesondere die international geläufige Unterscheidung von Palliative Care in eine Basiskompetenz aller Gesundheitsberufe und eine spezialisierte Disziplin fehlt in der Schweiz [10]. Das zeigt sich auch bei Kriterien und Charakteristika von Leistungen der spezialisierten Palliativversorgung wie 24-StundenHintergrunddienst, Zusammensetzung und Qualifikation der Fachteams und der Nutzung von Guidelines in der Praxis. Die Schweiz erfüllt hier internationale Vorgaben bspw. aus Kanada nicht [11]. Ein grosser Anteil der teilweise fehlenden oder widersprüchlichen Angaben (z. B. Integration in Palliativnetze, Selbstbewertung als spezialisierte Institution bei gleichzeitig fehlender Voraussetzung wesentlicher Strukturmerkmale) lässt sich sowohl auf die fehlenden Definitionen als auch auf fehlende Qualifikationspfade zurückführen.

Viele dieser Defizite haben Eingang gefunden in die «Nationale Strategie Palliative Care 2010-2012», die nicht zuletzt auf Grundlage dieser Erhebung im Oktober 2009 verabschiedet und definiert wurde [12]. Für die Zukunft braucht es vor allem einheitliche Definitionen, Qualitätsmerkmale und Bildungsangebote, die eine sinnvolle Integration in das bestehende Gesundheitssystem ermöglichen. Besonders wichtig ist hierbei die Kooperation mit den Grundversorgern [13].

\section{Für die Zukunft braucht es einheitliche Definitionen, Qualitätsmerkmale und Bildungsangebote, die eine Integration von Palliative Care in das bestehende Gesundheitssystem ermöglichen}

Die Vertreterinnen und Vertreter der hier befragten Institutionen befürworten die Integration von Palliative Care ins eigene Leitbild, besonders in Einrichtungen der Langzeitbetreuung. Eine besondere Qualität in der Betreuung von Menschen in dieser schwierigen Lebenslage vorweisen zu können, scheint für Image und Marketing wichtig zu sein, ebenso wie die Berücksichtigung der Patientenpräferenzen und der Einbezug der Angehörigen in Entscheidungsprozesse [9].

Nicht zuletzt aufgrund eines sehr uneinheitlichen Gebrauchs der Begriffe bestehen Unsicherheiten in der

\section{Schlussfolgerungen}

Das bestehende Angebot an Palliative Care in der Schweiz ist sehr heterogen, entbehrt einer gemeinsamen definitorischen Grundlage und ist regional sehr unterschiedlich ausgeprägt. Ein qualitativ hochstehendes Angebot an regional optimal vernetzter Palliative Care mit Grundversorgern und mobilen Diensten, Hospizen, Alters- und Pflegeheimen und Spitälern im Verbund sollte für die Zukunft eine wesentliche Alternative zum «Lebensende in der Institution» [14] werden. 
Die vorliegende Bestandesaufnahme für Palliative Care in der Grundversorgung und in spezialisierten Institutionen stellt eine Grundlage für die Weiterverfolgung dieser Entwicklung dar. Auch die Politik hat die Brisanz dieser Fragen erkannt. Es geht hierbei nicht um die Einführung neuer zusätzlicher Leistungen, sondern darum, dass Bund und Kantone Palliative Care in der Schweiz als festen Bestandteil des Gesundheitswesens verankern; dies über ein klares Profil von Palliative Care, Definitionen der Leistungen, kontinuierliche Information und Sensibilisierung der Bevölkerung sowie über weitere Schritte bei Finanzierung, Bildung und Forschung. Eine solche Integration hat in anderen Ländern gezeigt, dass nicht nur die Diskussionen über die Sterbehilfe eine Entkrampfung erfahren, sondern dass Mittel im Gesundheitswesen wesentlich prioritärer entsprechend den Wünschen der Patienten eingesetzt werden [15]. Die neue Nationale Strategie Palliative Care 2010-2012 [12] setzt für die kommenden Jahre die entsprechenden Schwerpunkte, um die Herausforderungen der Zukunft anzugehen.

Eine volle Version der Bestandesaufnahme [16] findet sich unter: www.palliative.ch/uni_pdf/bericht_ pallcare_survey08.pdf

\section{Literatur}

1 Bundesamt für Statistik: Szenarien zur Bevölkerungsentwicklung in der Schweiz 2005-2050, Neuchâtel 2006. www.bfs.admin.ch/bfs/portal/de/index/ themen/01/03/blank/key/intro.html

2 Jaccard Ruedin H, Weaver F. Ageing Workforce in an Ageing Society. Wieviele Health Professionals braucht das Schweizer Gesundheitssystem bis 2030? Careum Working Paper 1. Schweizerisches Gesundheitsobservatorium. August 2009.

3 Fischer S, Bosshard G, Zellweger U, Faisst K. Der Sterbeort: Wo sterben die Menschen heute in der Schweiz?. Z Gerontol Geriat. 2004,37:467-74.

4 WHO Europe: Palliative Care. The solid facts. 2004 www.euro.who.int/document/E82931.pdf

5 Eychmüller S, Raemy-Bass C. Bestandesaufnahme zur Situation von Palliative Care in der Schweiz. Infokara. 2001;1:23-6.
6 Centeno C et al. EAPC Task Force Development of Palliative Care in Europe. A Map of Palliative Care Specific Resources in Europe. 4th Research Forum of the EAPC. Venezia: 2006. www.eapcnet.org/ download/forTaskforces/DevelopTF-Map.pdf

7 Deutsche Hospiz-Stiftung. Was denken die Deutschen über Palliative Care? Emnid-Umfrage 2003. www.hospize.de/docs/stellungnahmen/14.pdf

8 Bundesamt für Gesundheit (BAG): Handlungsbedarf im Bereich Palliative Care. Schlussbericht Infras (Trageser J, von Stokar T, Stern S, Zandonella R). Zürich; Oktober 2009.

9 Kunz R. Individuelle Bedürfnisse - ganzheitliche Angebote. Betreuung von älteren Menschen in der letzten Lebensphase. In: Curaviva (Hrsg.): Orte des Lebens - Orte des Sterbens, 2008, S. 11-20.

10 National Health Service UK. Specialist Palliative Care www.ncpc.org.uk/download/policy/pbr/SPC_ HRGsv4.pdf

112001 Proposed Norms of Practice for Practice for Hospice Palliative Care (Canadian Palliative Care Association Standards Committee) www.chpca.net/

12 Bundesamt für Gesundheit (BAG) und Schweizerische Konferenz der kantonalen Gesundheitsdirektorinnen und -direktoren (GDK) (Hrsg). Binder J, von Wartburg L. Nationale Strategie Palliative Care 2010-2012. Bern; Oktober 2009.

13 Seematter-Bagnoud L et al. Angebot und Inanspruchnahme ambulanter medizinischer Dienstleistungen in der Schweiz - Prognosen bis 2030. Arbeitsdokument 33. Schweizerisches Gesundheitsobservatorium; 2008.

14 Hoepflinger F, Hugentobler V. Familiale, ambulante und stationäre Pflege im Alter. Perspektiven für die Schweiz. Buchreihe des Schweizerischen Gesundheitsobervatoriums. Bern; 2005.

15 van der Heide A et al. End-of-life practices in the Netherlands under the euthanasia act. N Engl J Med. 2007;356:1957-65. content.nejm.org/cgi/ reprint/356/19/1957.pdf

16 Eychmüller S, Schmid M, Müller M. Palliative Care in der Schweiz - Nationale Bestandesaufnahme 2008. Schlussbericht Projekt No OCS 01776-08-2005. Oncosuisse; Juni 2009. 